\title{
Modeling the acceleration field and objective lens for an aberration corrected photoemission electron microscope
}

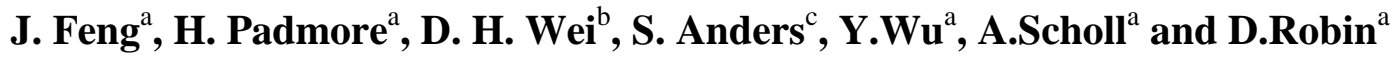 \\ ${ }^{a}$ ALS, Lawrence Berkeley National Laboratory, Berkeley, CA 94710 USA \\ ${ }^{b}$ SRRC, No.1 R\&D Rd. VI, Hsinchu 300, Taiwan \\ ${ }^{c}$ IBM, Almaden Research Center, 650 Harry Road, San Jose, CA 95120 USA
}

The modeling of the optical properties of the acceleration field and objective lens of a photoemission electron microscope is presented. Theory to calculate the aberrations of the extraction field was derived, and extended to include relativistic effects. An analysis of the microscopes electron optical performance and aberrations has been performed using an analytical model as well as a raytracing method. Raytracing has the flexibility needed for the assessment of aberrations where the geometry is too complex for analytical methods. This work shows that in the case of a simple PEEM front end of the acceleration gap and objective lens, the all orders raytracing and full analytical treatments agree to very high precision. This allows us now to use the raytracing method in situations where analytical methods are difficult, such as an aberration compensating electron mirror.

\section{Introduction}

The second generation photoemission electron microscope (PEEM2) at the ALS has reached its design goal and a spatial resolution of $20 \mathrm{~nm}$ has been achieved [1]. The resolution of the PEEM2 microscope is mainly limited by the aberrations of the 
acceleration field, the objective lens, and the diffraction at the back focal plane angle defining aperture and cannot be reduced by modifying the electrostatic lens design. Aberrations must be corrected in order to remove their deleterious effects on the imaging properties of the microscope for higher resolution applications. The most promising approach to an aberration corrected PEEM is the introduction of an electrostatic mirror into PEEM[2-4]. An electrostatic mirror, in principle, can be used for the simultaneous correction of chromatic and spherical aberrations. The design goal of an aberration corrected PEEM (called PEEM3 in this paper) is to obtain the highest throughput, at a resolution commensurate with the resolution determined by low energy electron scattering within a sample (typically $3 \mathrm{~nm}$ ).

A magnetic separator with very small aberration has being designed to direct the beam coming from the objective into the aberration correcting mirror, and then to redirect the beam back along the optical axis of the instrument. This separator has aberration of < $1 \mathrm{~nm}$ for the full secondary electron emission energy profile for a $20 \mathrm{kV}$ extraction field and an objective lens magnification of 20 . In order to design the correcting mirror that ultimately sets the resolution, a full description of the aberrations of the extraction field and objective lens are required.

\section{Model}

The first optical element of PEEM3 is a tetrode lens, which consists of the sample and a three-element unipotential lens. The sample is at negative potential of typically $5-30 \mathrm{kV}$ and acts as the cathode, the first electrode of the objective lens is at ground potential and acts as the anode. The extraction/objective lens must both accelerate the electrons emitted from the sample and form a magnified image for further magnification by other 
elements. Fig.1 shows an equipotential plot of the tetrode lens. The transverse displacement $\Delta \mathrm{r}$ of an object point caused by the chromatic and spherical aberrations of the acceleration field is given by [5]

$$
\begin{aligned}
& \Delta r=2 \rho L \sin \alpha\left[\frac{\frac{\rho_{0}}{\rho}\left(\sqrt{1 / \rho_{0}+1}-1\right)+\cos ^{2} \alpha}{\sqrt{1 / \rho_{0}+\cos ^{2} \alpha}}-\cos \alpha\right] \ldots \ldots(1) \\
& \rho=E / U
\end{aligned}
$$

where $\mathrm{L}$ is the distance between the sample and the first electrode of the objective lens, $\mathrm{E}$ is the electron energy, $\mathrm{U}$ is acceleration potential, and $\alpha$ is the emission angle of the electrons to the surface normal. Subscript 0 represents the peak of the electron energy distribution. For $\mathrm{E} / \mathrm{U}<<1$, an approximate formula based on eq.(1) can be derived [6]

$$
\Delta r=\frac{2 L}{U}\left(\sqrt{E_{0}}-\sqrt{E} \cos \alpha\right) \sqrt{E} \sin \alpha \ldots \ldots(2)
$$

Assuming that the diverging aperture lens is very weak so that the acceleration field can be simplified as a planar emission cathode, the aberration formulae for the acceleration field are as given in Eq.(1) and Eq.(2). However, due to the high resolution we ultimately desire, these formulae have to be modified to include the effect of relativity as follows;

$$
\begin{aligned}
& \Delta r=\frac{c L P \sin \alpha}{e U}\left[\arccos h\left(\frac{\frac{e U}{c}+\sqrt{m_{e}^{2} c^{2}+P^{2}}}{\sqrt{m_{e}^{2} c^{2}+P^{2} \sin ^{2} \alpha}}\right)-\arcsin h\left(\frac{P \cos \alpha}{\sqrt{m_{e}^{2} c^{2}+P^{2} \sin ^{2} \alpha}}\right)\right] \\
& +\frac{L \sin \alpha}{\sqrt{\cos ^{2} \alpha+\frac{U}{E}}} \frac{c P_{0} \sqrt{1+\frac{U}{E_{0}}}}{e U}\left[\operatorname{arccosh}\left(\frac{\frac{e U}{c}+\sqrt{m_{e}^{2} c^{2}+P_{0}^{2}}}{m_{e} c}\right)-\arcsin h\left(\frac{P_{0}}{m_{e} c}\right)\right] \ldots
\end{aligned}
$$


where $\mathrm{c}$ is the speed of light, e is the electron charge, me is the electron mass, $\mathrm{P}$ is the momentum.

The spherical aberration coefficient $\mathrm{C}_{\mathrm{s}}$ and chromatic aberration coefficient $\mathrm{C}_{\mathrm{c}}$ of a unipotential lens can be expressed as [7]

$$
\begin{aligned}
& C_{S}=\int_{Z_{0}}^{Z_{1}}\left\{\frac{1}{16}\left[\frac{5}{4}\left(\frac{U^{\prime \prime}}{U}\right)^{2}+\frac{5}{24}\left(\frac{U^{\prime}}{U}\right)^{4}\right] r^{4}+\frac{14}{48}\left(\frac{U^{\prime}}{U}\right)^{3} r^{\prime} r^{3}-\frac{3}{32}\left(\frac{U^{\prime}}{U}\right)^{2} r^{2} r^{2}\right\} \sqrt{U_{0} / U} d z \ldots \ldots(4) \\
& C_{c}=\int_{Z_{0}}^{Z_{1}}\left(\frac{U^{\prime}}{2 U} r r^{\prime}+\frac{U^{\prime \prime}}{4 U} r^{2}\right) \sqrt{U_{0} / U} d z \ldots \ldots(5)
\end{aligned}
$$

where $\mathrm{r}$ and $r^{\prime}$ are the electron displacement and angle along path direction, $\mathrm{U}$ is the local potential, and $U^{\prime}, U^{\prime \prime}$ are first and second derivative of potential along the beam direction, respectively. $\mathrm{U}_{0}=\mathrm{E}_{0} / \mathrm{e}$ is related to the electron energy $\mathrm{E}_{0}$, and all aberration coefficients are referred to the object side.

Eqs.(4)-(5) were originally derived by Scherzer [8]. Since they don't contain any undesirable higher derivatives of the axial potential and flux density distributions, they are the form most commonly used for practical calculations of the electrostatic lens aberration coefficients. The importance of these equations is that they allow one to calculate aberrations that describe the performance of an optical system when transmitting a range of angles and energies, even though they are derived from the calculation of one trajectory; this is a tremendous simplification over raytracing where each electron is treated individually. The restriction is in the complexity of system that can be handled, limited by the presence of higher order aberrations.

Image formation in PEEM utilizes secondary, photo and Auger electrons emitted from the sample by incident X-ray. The energy distribution of emitted secondary electrons is well modeled by a function of the form $\mathrm{E} /\left(\mathrm{E}+\mathrm{W}_{\mathrm{f}}\right)^{4}$, where $\mathrm{E}$ is the electron energy and 
$\mathrm{W}_{\mathrm{f}}$ is the work function of the materials [1]. The probability per unit solid angle and unit energy of an electron being emitted with $(\alpha, E)$ is

$$
\begin{aligned}
& w(\alpha, E)=\frac{E\left(E+W_{f}\right)^{-4} \cos \alpha}{2 \pi \int_{0}^{\infty} \int_{0}^{\pi / 2} E\left(E+W_{f}\right)^{-4} \cos \alpha \sin \alpha d \alpha d E} \\
& =\frac{E\left(E+W_{f}\right)^{-4} \cos \alpha}{\pi /\left(6 W_{f}^{2}\right)} \ldots . .(6)
\end{aligned}
$$

To reduce aberrations to an acceptable level, an aperture is put in the back focal plane of the acceleration+objective lens. Watt, et al., [6] derived an equation to express how this aperture limits the transmission of a ray with angle $\alpha$ and energy $\mathrm{E}$

$$
E \leq \frac{a^{2} U}{\left(f_{i}^{*}\right)^{2} \sin ^{2} \alpha}
$$

where a is the aperture radius, $\mathrm{U}$ is the sample potential, and $f_{i}^{*}$ is the image side focal length of the combined acceleration and objective lens.

The transmission can then be derived from the following integral, which, surprisingly, can be evaluated in simple closed form

$$
\begin{aligned}
& T=2 \pi \int_{0}^{\pi / 2} \int_{0}^{E(\alpha)} \frac{E\left(E+W_{f}\right)^{-4} \cos \alpha \sin \alpha d \alpha d E}{\pi /\left(6 W_{f}^{2}\right)} \\
& =1-\frac{1}{\left[1+\left(\frac{a}{f_{i}^{*}}\right)^{2} \frac{U}{W_{f}}\right]^{2}} \ldots . .(8)
\end{aligned}
$$

Eq.(8) shows that the transmission is a function of the aperture size, image focal length, sample potential and work function. Higher sample potential or a larger aperture size gives a higher transmission, whereas, longer focal length or larger work function gives a lower transmission. 


\section{Results and discussion}

Fig.2 gives the effect of relativity on the aberration of the acceleration field. A $4 \mathrm{ev}$ work function is used for the calculation. The electron energy range is from $0 \mathrm{eV}$ to $20 \mathrm{eV}$, with a takeoff angle is from 0 degree to 90 degree. Generally speaking, the aberration of the acceleration field without relativity is underestimated, but is only half of our design resolution for extreme rays in this case, and can be ignored in general.

Fig.3 shows how the electron energy distributions evolve with 500, 200, 100, 50 microns aperture size in diameter. It was calculated by an all-orders trajectory ray tracing method using custom developed internal electron optical code and SIMION 7.0 [9]. Single points in the figure represents one electron. There are in total 8100 electrons. The aperture is located at the back focal plane of the acceleration/objective lens. The energy distribution becomes narrower and the intensity becomes smaller with decreasing aperture size, but higher energy electron with a smaller angle can still pass through the aperture. Although a back focal plane aperture will dramatically improve resolution, due to a reduced angle (hence reduced spherical aberration) and reduced energy range (hence reduced chromatic aberration), the minimum size is limited by electron diffraction. In general, transmission through this small aperture is small. For example, for the smallest aperture used in PEEM2 $(12 \mu \mathrm{m})$, the transmission is around 3\%. In the PEEM3 system, we aim to substantially reduce chromatic and spherical aberrations using an electron mirror system and much higher transmission at higher resolution should be achievable.

The transmission and resolution of the PEEM3 front end are presented in fig.4. Fig.4a) shows the transmission as a function of aperture size for a $4 \mathrm{eV}$ work function sample at 20kV. Fig.4b) shows the individual aberrations (the acceleration field aberrations, and the contribution of spherical and chromatic aberrations calculated from eqs.(3)-(5) ) to the 
total resolution. The excellent agreement over the large range of apertures provides convincing evidence for the accuracy of our all orders raytracing method and analytical method. The flexibility of the computational approach is allowing us to rapidly optimize and characterize the acceleration gap/objective lens system so that we can optimize the design of the aberration compensating mirror.

\section{Acknowledgements}

We have benefited greatly from conversations with M.Marcus, H.Ohldag, R.Duarte, R.Schlueter, K.Nicholas, A.Doran, E.Bauer and M.R.Scheinfein. This work was supported by the U.S. Department of Energy Office of Basic Energy Science, under contract No.DE-AC03-76sF00098.

\section{References}

[1] S. Anders, H. Padmore et al, Rev. Sci. Instrum. 70 (1999) 3973.

[2] G. F. Rempfer, J. Appl. Phys. 67, (1990) 6027.

[3] Z. Shao and X. D. Wu, Optik 84, (1990) 51.

[4] R. Fink, et al , J. Electron Spectroscopy Relat. Phenom. 84, (1997) 231.

[5] E.Bauer, Ultramicroscopy 17, 51(1985)

[6] R. N. Watts, S. Liang, Z. H. Levine, T. B. Lucatorto, F. Polack, and M. R. Scheinfein, Rev. Sci. Instrum. 68, 3464 (1997).

[7] M.Szilagyi, Electron and ion optics, Plenum Pres, New York 1988

[8] O.Scherzer, Über einige Fehler von Elektronenlinsen, Z.Phys. 101, 593(1936)

[9] D.A. Dahl, J. E. Dlmore, and A. D. Appelhans, Rev. Sci. Instrum. 61, 607(1990) 


\section{Figure captions}

Fig.1 Field contour plot of tetrode lens. The equipotential lines are $1250 \mathrm{~V}$ apart.

Fig.2 The effect of relativity on the aberrations of the acceleration field

Fig.3 Energy distributions as function of the back focal plane aperture

Fig.4 Resolution and transmission as a function of aperture size (diameter) 


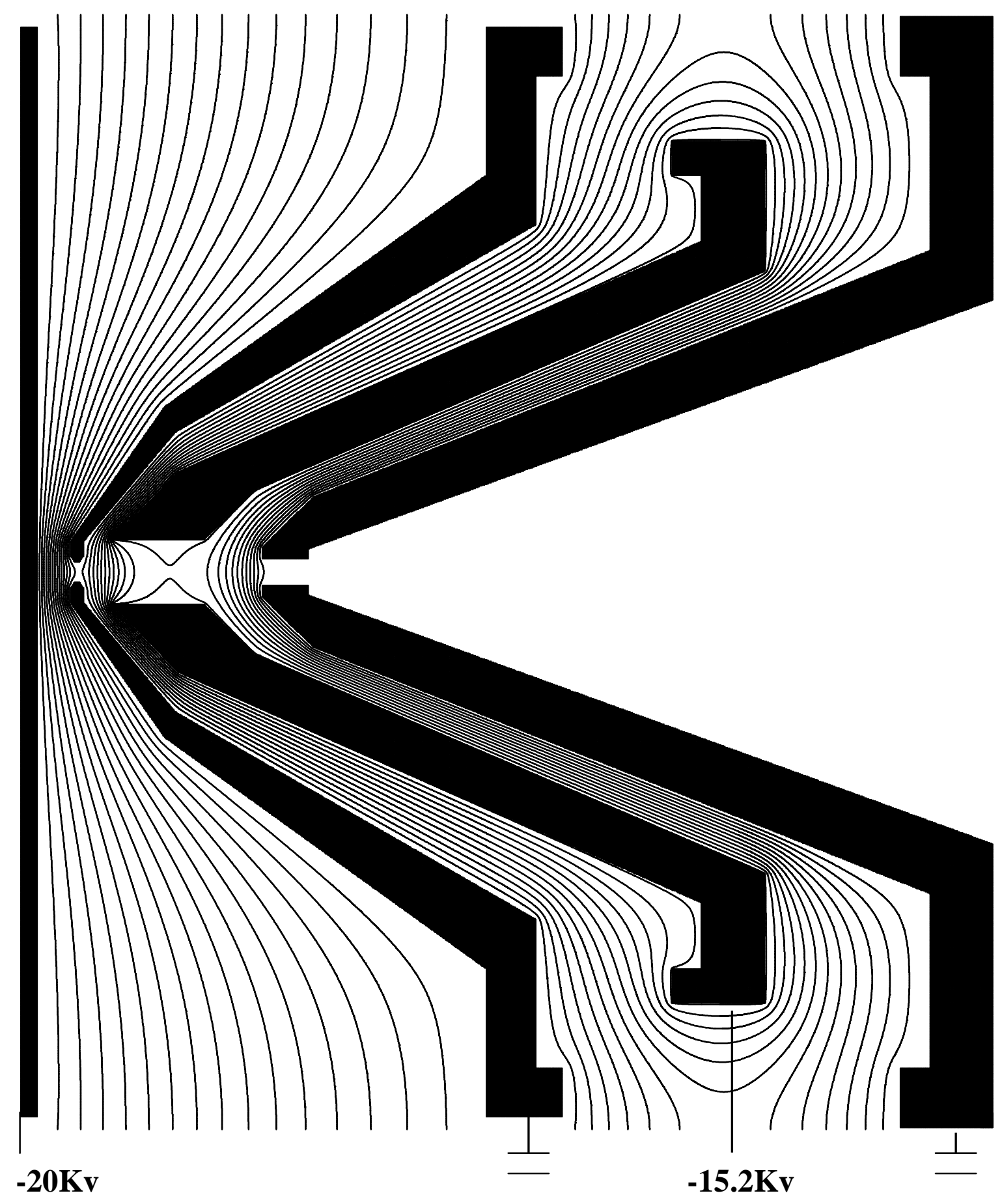

Fig.1 


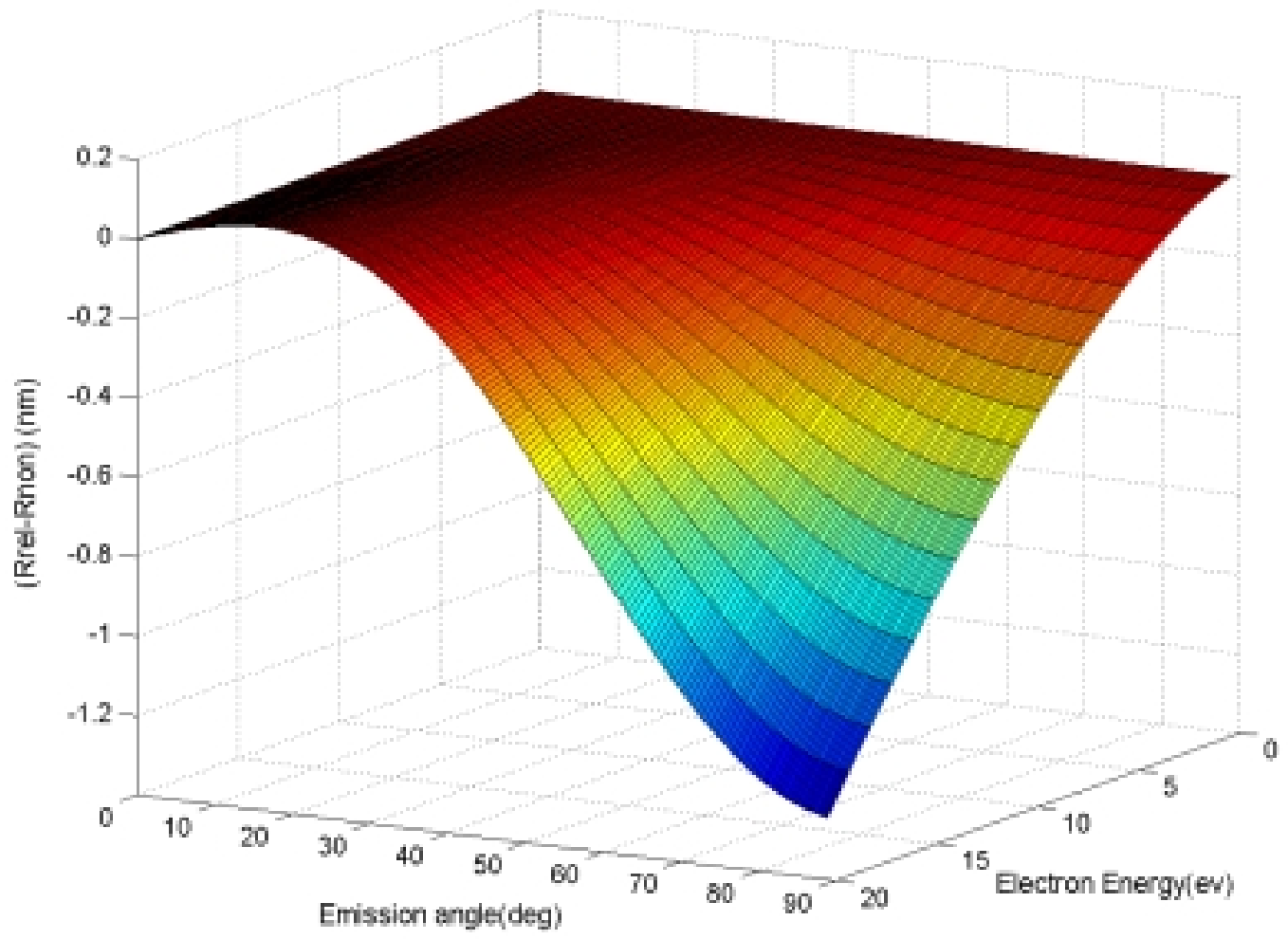

Fig.2 

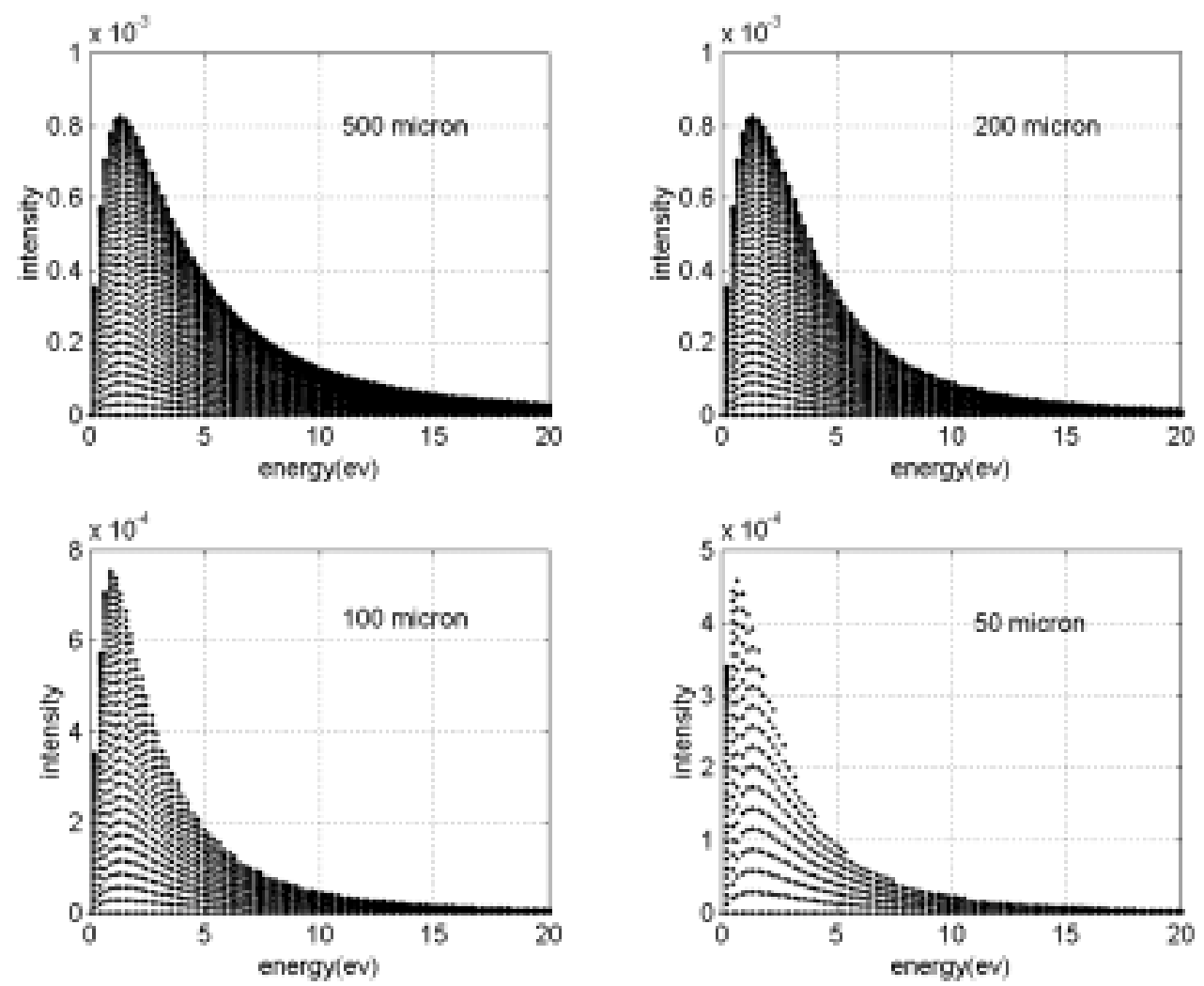

Fig. 3 


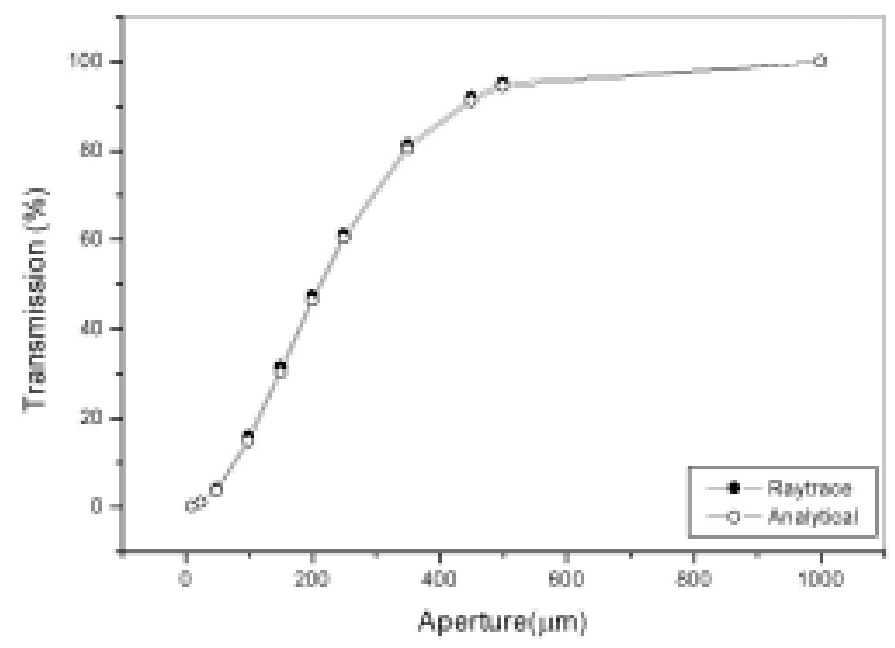

(a)

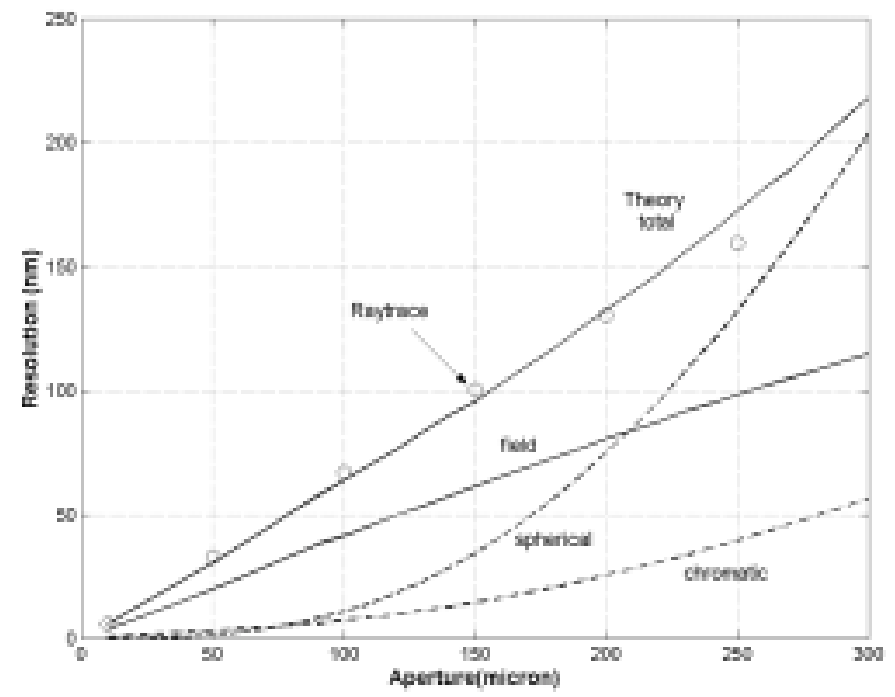

(b)

Fig.4 